\title{
Estimación De Los Residuos Forestales En Los Aserraderos De Tres Cantones, Zona 3 Interandina, Ecuador
}

\author{
Miguel Angel Guallpa Calva \\ Sonia Carmita Rosero Haro \\ Galo Briam Montenegro Córdova
}

Docentes Investigadores, Facultad de Recursos Naturales,

Escuela Superior Politécnica de Chimborazo, Riobamba, Ecuador

Darío Javier Quinchuela Guamán

Graduado de la Escuela de Ingeniería Forestal,

Escuela Superior Politécnica de Chimborazo, Riobamba, Ecuador

Doi: 10.19044/esj.2018.v14n30p228 URL:http://dx.doi.org/10.19044/esj.2018.v14n30p228

\begin{abstract}
This paper focuses on the current situation of the availability and use of forestry waste generated in sawmills of the Ecuadorian interandina zone 3. It specifically highlights information about different types of waste, its volume, sales price, and final location. Through the stratification of the sample, 38, 25 and 19 surveys were carried out with loggers from the cantons of Riobamba, Ambato, and Latacunga with a level of reliability of $95 \%$. It was stated that the most common waste are sawdust, shavings, and wood scrap. The monthly production average is $2.92,1.64$, and 2.74 tons of waste in the first village. $31.6 \%, 40 \%$, and $31.6 \%$ of sawmills in Riobamba, Ambato and Latacunga sells from 6.1-8, 4.1-6, and 8.1-10 dollars per ton respectively. There was highly significant differences in the sale prices $(p<0,0001)$ of the by product in the sawmills of the three villages. The evidence in Ambato is different and in contrast with sawmills in Riobamba and Latacunga which shows a similar behavior in selling forestry waste periodically in the stages of primary and secondary transformation. This, therefore, is in a bid to take advantage of the opportunities for the development of new enterprises.
\end{abstract}

Keywords: Sawmills, forest residuals, primary and secondary transformation, area 3 interandina

\section{Resumen}

El estudio evalúa la situación actual sobre la disponibilidad y usos de los residuos forestales generados en los aserraderos de la zona 3 InterandinaEcuador con la búsqueda de información sobre el tipo de residuo, su volumen, 
precio de venta y destino final. Mediante la estratificación de la muestra, se realizó 38, 25 y 19 encuestas a los madereros de los cantones Riobamba, Ambato y Latacunga con un nivel de confiabilidad del 95\%. Se determinó que los principales desechos son: aserrín, viruta y retazos. La producción promedio mensual reportó valores de 2.92, 1.64, y 2.74 toneladas de residuos cantón $^{-1}$. El 31.6\%, 40\%, 31.6\% de los aserraderos de los cantones Riobamba, Ambato y Latacunga venden de 6.1-8, 4.1-6, 8.1-10 dólares tonelada ${ }^{-1}$ respectivamente. Existen diferencias altamente significativas en el precio de venta $(\mathrm{p}<0,0001)$ de los subproductos en los aserraderos de los tres cantones, resultando diferente en Ambato, en contraste con los aserraderos de Riobamba y Latacunga los cuales mostraron un comportamiento similar al momento de vender los residuos forestales para la producción agropecuaria, tableros, ladrilleras y energía. Se sugiere evaluar periódicamente el volumen y disponibilidad de residuos madereros en las fases de transformación primaria y secundaria a fin de aprovechar las oportunidades para el desarrollo de nuevos emprendimientos.

Palabras clave: Aserraderos, residuos forestales, transformación primaria y secundaria, Zona 3 interandina

\section{Introducción}

El sector forestal formal en el mundo emplea a unos 13,2 millones de personas, y al menos otros 41 millones trabajan en el sector informal, estas actividades forestales no suele registrarse en las estadísticas nacionales, pero las estimaciones presentadas por el informe de las Naciones Unidas muestran que en las regiones menos desarrolladas unos 840 millones de personas, es decir, el $12 \%$ de la población mundial, recogen combustible de madera y carbón vegetal para su uso directo (FAO, 2014, p. 20). En Ecuador en 2011 la producción fue de 7 millones de $\mathrm{m}^{3}$ de madera en rollo, $519 \mathrm{mil} \mathrm{m}^{3}$ de madera aserrada, $882 \mathrm{mil} \mathrm{m}^{3}$ de tableros de madera, 240 mil toneladas de papel y cartón, 150 mil toneladas de papel recuperado, 2 mil toneladas de pulpa de madera y 16 mil toneladas de pulpa de otras fibras. Las industrias forestales del Ecuador producen cantidades relevantes de madera aserrada, la cual registra como el producto con el mayor crecimiento con $11 \%$ en comparación al año 2010, seguido de madera en rollo con $1 \%$. Los demás productos no registran crecimiento (PRO ECUADOR, 2013).

La leña y el carbón figuran en la categoría de subproductos madereros, constituyéndose como una fuente de ingresos para las comunidades campesinas, además de material energético de bajo costo (MAE, 2011, p. 13). Sin embargo no se considera, la alta cantidad de residuos que provienen tanto del aserradero y la transformación secundaria de la madera. En la industria maderera se transforma la troza en madera aserrada y se puede obtener otros 
productos tales como: costeras, aserrín, residuos sólidos o corteza (Vignote \& Martínez, 2006). Generalmente estos residuos son utilizados como materia prima para la industria de celulosa y de tableros, la generación de energía y para usos agropecuarios (Manzanares, Velázques, \& Guyat, 2007).

La escasa información sobre la cantidad de residuos que se generan en los aserraderos de la Zona 3 interandina del Ecuador, representa una limitante que influye en los costos de manipulación y su eliminación, mismos que ocasionan riesgos ambientales e industriales al sector. Aspectos que impiden un aprovechamiento eficiente, considerando que su volumen es de hasta el $60 \%$ con respecto a la materia prima de entrada (madera en trozas) en una instalación convencional (Chacón, 2012).

Por lo expuesto, su utilización puede convertirse en una potencial alternativa de materia prima para el desarrollo de la industria en la región interandina Zona 3, con la implementación de nuevas tecnologías que contribuyan en el control ambiental. Este tipo de estrategias están basadas en la llamada concentración vertical, los residuos de una producción constituyen insumos para otras líneas de flujo, propiciando así el reciclado e incrementando el valor agregado del producto o generando ingresos económicos por la venta de los mismos (Bequete, 1996).

A partir de este diagnóstico, se levantó la información considerando los parámetros oferta, uso actual y precios de venta. El objetivo del presente estudio fue tipificar los principales residuos generados en el sector $\mathrm{y}$ cuantificar su volumen. Con ello se dispone de información referencial sobre la situación de estos subproductos de aserraderos en la zona en estudio a fin de propiciar el uso integrado de ciertos subproductos de la madera, actualmente no aprovechados en su totalidad a nivel de aserraderos o en los encadenamientos que generen oportunidad de ser comercializados a empresas con capacidad de industrialización (Secretaría Nacional de Planificación y Desarrollo, 2013).

\section{Materiales y Métodos}

La investigación se realizó en los aserraderos localizados en los cantones: Riobamba, Ambato y Latacunga. Geográficamente los sitios se insertan en el entorno a los puntos de referencia: UTM Zona 17S Datum WGS 84 (Tabla 1).

Tabla 1. Ubicación geográfica de los cantones de implementación del estudio

\begin{tabular}{|l|c|c|c|}
\hline \multicolumn{1}{|c|}{ Cantones } & X & Y & Altitud (msnm) \\
\hline Riobamba & 755455 & 9817613 & 2750 \\
\hline Ambato & 768725 & 9872134 & 2577 \\
\hline Latacunga & 768725 & 9913742 & 2750 \\
\hline
\end{tabular}

En la investigación se aplicaron los métodos: documental y de campo, definidas para la caracterización de los aserraderos. 
El muestreo de las variables cuantitativas y cualitativas tuvo lugar el primer semestre del año 2014. Datos utilizados para realizar el análisis descriptivo y cálculo de la prueba de Kruskal Wallis para la variable precio de venta tonelada ${ }^{-1}$ de residuo en relación a los cantones en los cuales se realizó el estudio.

Los datos básicos a partir del cual se obtiene el volumen de residuos madereros fueron la producción y el consumo (toneladas). Los propietarios de aserraderos facilitaron la información de la producción semanal, quincenal y mensual, más no todos el consumo en los periodos indicados. Por lo que se procedió a calcular el promedio mensual por cantón de esta variable.

En la determinación del análisis de la oferta se trabajó de la siguiente manera:

\section{Segmentación del Mercado}

Los aserraderos que generan los residuos, se sitúan en los cantones Riobamba, Ambato y Latacunga.

\section{Universo}

El universo de estudio es de 104 potenciales ofertantes de residuos de la madera en la zona 3 Interandina según datos facilitados en cada oficina técnica del MAE, en el año 2014, se registraron 50 aserraderos en Riobamba, 32 en Ambato y 24 en Latacunga.

\section{Muestra}

La muestra se calculó en base a la fórmula de Canavos (Pazmiño, 1997).

Donde:

$$
\mathrm{n}=\frac{\mathrm{N} * \mathrm{p} * \mathrm{q}}{(\mathrm{N}-1) *\left(\frac{\mathrm{e}}{\mathrm{z}}\right)^{2}+(\mathrm{p} * \mathrm{q})}
$$

n: tamaño de la muestra

$\mathrm{N}$ : universo de estudio

e: margen de error o precisión admisible (5\%)

z: nivel de confianza $(1,96)$

p: probabilidad de ocurrencia (0.5)

q: probabilidad de no ocurrencia (0.5)

$$
\begin{gathered}
\mathrm{n}=\frac{104 *(0.5) *(0.5)}{(104-1) *\left(\frac{0.05}{1.96}\right)^{2}+(0.5 * 0.5)} \\
\mathrm{n}=\frac{26}{0,32} \\
\mathrm{n}=82,01
\end{gathered}
$$




\section{Estratificación de la muestra}

En la estratificación se consideró la composición en base a la cantidad de aserraderos existentes en cada cantón (Tabla 2).

Tabla 2. Población de aserraderos cantón ${ }^{-1}$

\begin{tabular}{|l|c|}
\hline \multicolumn{1}{|c|}{ Aserraderos cantón } & Número de aserraderos \\
\hline Riobamba & 48 \\
\hline Ambato & 32 \\
\hline Latacunga & 24 \\
\hline Total & 104 \\
\hline
\end{tabular}

\section{Factor de estratificación (f)}

Donde:

n: tamaño de la muestra

$\mathrm{N}$ : universo de estudio

$$
f=\frac{\mathrm{n}}{N}=\frac{82}{104}=0,7885
$$

La segmentación de la muestra de los aserraderos por cantón (Tabla 3 ), se determinó al multiplicar la cantidad de aserraderos de cada cantón por el factor de estratificación $(0,7885)$.

Tabla 3. Segmentación de la muestra de los aserraderos cantón ${ }^{-1}$

\begin{tabular}{|l|c|c|}
\hline Aserraderos cantón & $\begin{array}{c}\text { Aserraderos de cada cantón } \\
\text { por el factor de estratificación }\end{array}$ & $\begin{array}{c}\text { Número de encuestas aplicadas } \\
\text { en los aserraderos cantón }^{-1}\end{array}$ \\
\hline Riobamba & $48 \times 0,7885$ & 38 \\
\hline Ambato & $32 \times 0,7885$ & 25 \\
\hline Latacunga & $24 \times 0,7885$ & 19 \\
\hline Total & & 82 \\
\hline
\end{tabular}

\section{Análisis estadístico}

Se elaboró tablas de frecuencias para el proceso de las variables cualitativas y cuantitativas. Para el análisis de los datos se utilizó, hoja de cálculo de Excel y el software Infostat (Balzarini et al., 2008).

\section{Resultados y Discusión}

\section{Frecuencia de residuos que se generan por cantón}

La generación de residuos de aserrín, viruta y retazos, resulto similar en los tres cantones, ocupando el primer lugar el 45,24\% de aserrín en Latacunga, el 42,11\% de viruta en Ambato y el 15,12\% de retazos corresponde a Riobamba (Tabla 4) 
Tabla 4. Generación de residuos en cada cantón

\begin{tabular}{|c|c|c|c|c|c|c|c|c|}
\hline \multirow{2}{*}{ Cantones } & \multicolumn{2}{|c|}{ Aserrín } & \multicolumn{2}{|c|}{ Viruta } & \multicolumn{2}{|c|}{ Retazos } & \multirow{2}{*}{$\begin{array}{l}\text { Frec } \\
\text { abs total }\end{array}$} & \multirow{2}{*}{$\begin{array}{l}\text { Frec rel } \\
\text { total }\end{array}$} \\
\hline & Frec abs & Frec rel & Frec abs & Frec rel & Frec abs & Frec rel & & \\
\hline Riob & 38 & 44,19 & 35 & 40,70 & 13 & 15,12 & 86 & 100 \\
\hline Ambato & 25 & 43,86 & 24 & 42,11 & 8 & 14,04 & 57 & 100 \\
\hline Latacunga & 19 & 45,24 & 17 & 40,48 & 6 & 14,29 & 42 & 100 \\
\hline
\end{tabular}

Frecuencia absoluta (Frec abs), Frecuencia relativa (Frec rel).

Los resultados obtenidos a nivel de aserraderos indican que fundamentalmente se generan; aserrín, viruta y retazos. La escasa información disponible y el desconocimiento de la reutilización y aprovechamiento de los residuos de madera no permite utilizar los mismos para plantear nuevas oportunidades de negocio y manejo adecuado para reducir riesgos ambientales y cumplir con normas legales establecidas (Amaya, 2015).

En este contexto existen una serie de vías para el aprovechamiento de los residuos de aserraderos y especialmente el aserrín, el cual es frecuentemente utilizado para la producción de papel, tableros, elaboración de abonos o sustratos, pero en los países que no cuentan con estas tecnologías su utilización como combustible (Kollmann, 2001). En cambio Chacón (2012), sostiene que el aserrín no puede aprovecharse en las industrias de tableros, una alternativa de aprovechamiento es la peletización.

\section{Frecuencia de abastecimiento de los residuos}

La tabla 5 indica que la mayor cantidad de residuos se generan para la venta en un periodo de tiempo de un mes con $47.37 \%, 52.63 \%$ y el $68 \%$, mismos que corresponden a los cantones: Riobamba, Ambato y Latacunga respectivamente.

Tabla 5. Porcentaje de frecuencia de residuos en los aserraderos de cada cantón

\begin{tabular}{|l|c|c|c|}
\hline Cantón & Periodo & Frec abs & Frec rel (\%) \\
\hline Riobamba & Semanal & 11 & 28,95 \\
\hline Riobamba & Quincenal & 9 & 23,68 \\
\hline Riobamba & Mensual & 18 & 47,37 \\
\hline Total & & 38 & 100,00 \\
\hline Ambato & Semanal & 8 & 42,11 \\
\hline Ambato & Quincenal & 1 & 5,26 \\
\hline Ambato & Mensual & 10 & 52,63 \\
\hline Total & & 19 & 100,00 \\
\hline Latacunga & Semanal & 4 & 16,00 \\
\hline Latacunga & Quincenal & 4 & 16,00 \\
\hline Latacunga & Mensual & 17 & 68,00 \\
\hline Total & & 25 & 100,00 \\
\hline
\end{tabular}

Frecuencia absoluta (Frec abs), Frecuencia relativa (Frec rel). 


\section{Nivel de frecuencia en la generación de residuos forestales por cantón}

En el cantón Latacunga se produce el mayor porcentaje de residuos semanal con el 31,6\% que corresponde a una tonelada. En el cantón Riobamba por quincena, se tiene el 23,7\% (1 tonelada). El cantón Ambato tiene el 68\% mensual equivalente a una tonelada de residuos (Tabla 6).

En sentido general el volumen de producción mensual promedio de residuos forestales estimados es de 1.64, 2.92, y de 9.32 toneladas que corresponden a los cantones: Ambato, Riobamba y Latacunga respectivamente (Anexo 1). Cuya producción real de residuos de productos madereros, es heterogénea de una instalación a otra y dependiendo de las propiedades de la madera, el tipo de funcionamiento, mantenimiento de la industria elaboradora (Castillo, 2007).

Tabla 6. Porcentaje de aserraderos que generan entre 1 y 4 toneladas de residuos forestales por cantón

\begin{tabular}{|c|c|c|c|c|}
\hline & Rendimiento & Riobamba & Ambato & Latacunga \\
\hline Frecuencia & $(\mathrm{t})$ & $(\%)$ & $(\%)$ & $(\%)$ \\
\hline \multirow{4}{*}{ Semanal } & 1 & 18,4 & 16,0 & 31,6 \\
\cline { 2 - 5 } & 2 & 5,3 & & 10,5 \\
\cline { 2 - 5 } & 3 & 2,6 & & \\
\cline { 2 - 5 } & 4 & 2,6 & & 5,3 \\
\hline \multirow{3}{*}{ Quincenal } & 1 & 23,7 & 16,0 & 52,6 \\
\hline \multirow{3}{*}{ Tensual } & 1 & 42,1 & 68,0 & \\
\cline { 2 - 5 } & 3 & 2,6 & & 100 \\
\cline { 2 - 5 } & 4 & 2,6 & & 100 \\
\hline
\end{tabular}

Tonelada (t)

\section{Precios por tonelada de residuos}

El 31,6\% de los aserraderos del cantón Riobamba venden la tonelada de residuos entre $\$ 6,1$ a 8 dólares, en Ambato el $40 \%$ venden a precios que oscilan entre $\$ 4,1$ a 6 . En cambio en Latacunga el 31,6\% de aserraderos venden a precios entre $\$ 8,1$ a 10 (Tabla 7).

Tabla 7. Precios por tonelada y frecuencia de aserraderos por cantón en estudio.

\begin{tabular}{|c|c|c|c|c|c|c|}
\hline Cantones & \multicolumn{2}{|c|}{ Riobamba } & \multicolumn{2}{c|}{ Ambato } & \multicolumn{2}{c|}{ Latacunga } \\
\hline $\begin{array}{c}\text { Rango } \\
\text { (dólares) }\end{array}$ & Frec abs & $\begin{array}{c}\text { Frec rel } \\
(\boldsymbol{\%})\end{array}$ & Frec abs & $\begin{array}{c}\text { Frec rel } \\
(\boldsymbol{\%})\end{array}$ & Frec abs & $\begin{array}{c}\text { Frec rel } \\
(\boldsymbol{\%})\end{array}$ \\
\hline $1-2$ & 0 & 0,0 & 5 & 20,0 & 0 & 0,0 \\
\hline $2,1-4$ & 1 & 2,6 & 4 & 16,0 & 1 & 5,3 \\
\hline $4,1-6$ & 6 & 15,8 & 10 & 40,0 & 3 & 15,8 \\
\hline $6,1-8$ & 12 & 31,6 & 4 & 16,0 & 4 & 21,1 \\
\hline $8,1-10$ & 10 & 26,3 & 0 & 0,0 & 6 & 31,6 \\
\hline $10,1-12$ & 7 & 18,4 & 2 & 8,0 & 3 & 15,8 \\
\hline $12,1-14$ & 0 & 0,0 & 0 & 0,0 & 0 & 0,0 \\
\hline $14,1-16$ & 0 & 0,0 & 0 & 0,0 & 2 & 10,5 \\
\hline $16,1-18$ & 2 & 5,3 & 0 & 0,0 & 0 & 0,0 \\
\hline Total & 38 & 100,0 & 25 & 100,0 & 19 & 100,0 \\
\hline
\end{tabular}

Frecuencia absoluta (Frec abs), Frecuencia relativa (Frec rel). 
Los precios de los residuos forestales de aserradero en los tres cantones, zona 3 interandina-Ecuador muestran un comportamiento heterogéneo y no forman parte de un mercado bien establecido y maduro como se advierte en un estudio similar del uso de la biomasa de origen forestal realizado en Argentina (Uasuf \& Hilbert, 2012).

\section{Aplicación de la prueba Kruskal Wallis al precio de venta tonelada-1}

A un nivel del 5\%, la prueba en mención mostró que existen diferencias altamente significativas en la mediana del precio de venta de los residuos en los aserraderos de los tres cantones motivo de estudio ( $p<0,0001$, Tabla 8).

Tabla 8. Prueba de Kruskal Wallis aplicada a la variable precio de venta tonelada ${ }^{-1}$

\begin{tabular}{|c|c|c|c|c|c|c|}
\hline Cantón & $\mathbf{N}$ & Medias & D.E & Medianas & H & p \\
\hline Riobamba & 38 & 9,20 & 3,07 & 9,00 & 24,19 & $<0,0001$ \\
\hline Ambato & 25 & 5,28 & 2,83 & 5,00 & & \\
\hline Latacunga & 19 & 9,32 & 3,06 & 10,00 & & \\
\hline
\end{tabular}

El precio de venta de los residuos es diferente al menos en un cantón (Ambato; A), en contraste con los aserraderos de los cantones Riobamba y Latacunga los cuales mostraron un comportamiento similar al momento de vender los residuos forestales (Tabla 9).

Tabla 9. Rangos según prueba de Kruskal Wallis

\begin{tabular}{|c|c|}
\hline Cantones & Rangos \\
\hline Ambato & $21,98 \quad \mathrm{~A}$ \\
\hline Riobamba & $49,70 \quad \mathrm{~B}$ \\
\hline Latacunga & $50,79 \quad \mathrm{~B}$ \\
\hline
\end{tabular}

Medias seguidas por la misma letra, no presentan diferencias estadísticas $(p>0.05)$

\section{Preferencias de especies utilizadas}

La especie más utilizada en los tres cantones es eucalipto (Eucalyptus globulus) con un rango del 34 al 41.03\%, seguida por pino (Pinus radiata) con el 8,62\% al 30,77\%. Las especies menos utilizadas son: colorado (Guarea purusana), teca (Tectona grandis), y canelo (Ocotea sp.) con el $1 \%$ en Riobamba, y en Ambato mostraron un comportamiento similar; colorado (Guarea purusana), pigue (Pollalesta discolor), pilche (Vantanea spp.) y mascarey (Hyeronima macrocarpa) con el $1.72 \%$, en cambio en Latacunga con el $2.56 \%$ se reportó para colorado (Guarea purusana), mascarey (Hyeronima macrocarpa), chanul (Humiriastrun procerum), y chuncho (Cedrelinga cateneiformis) (Tabla10). 
Tabla 10. Porcentaje del nivel de preferencia al momento de adquirir los residuos de aserraderos

\begin{tabular}{|l|l|c|c|c|}
\hline \multirow{2}{*}{$\begin{array}{c}\text { Nombre } \\
\text { común }\end{array}$} & \multirow{2}{*}{ Nombre científico } & Riobamba & Ambato & Latacunga \\
\cline { 3 - 5 } & & $(\%)$ & $(\%)$ & $(\%)$ \\
\hline Eucalipto & Eucalyptus globulus & 34,0 & 34,48 & 41,03 \\
\hline Pino & Pinus radiata & 26,0 & 8,62 & 30,77 \\
\hline Ciprés & Cupressus macrocarpa & 5,0 & 3,45 & 12,82 \\
\hline Colorado & Guarea purusana & 1,0 & 1,72 & 2,56 \\
\hline Motilón & Hyeronima alchorneoides & & 15,52 & \\
\hline Pigue & Pollalesta discolor & 5,0 & 1,72 & \\
\hline Pilche & Vantanea spp. & & 1,72 & \\
\hline Nogal & Juglans neotropica & 2,0 & & \\
\hline Teca & Tectona grandis & 1,0 & & \\
\hline Mascarey & Hyeronima macrocarpa & 2,0 & 1,72 & 2,56 \\
\hline Copal & Dacryodes peruviana & 2,0 & & \\
\hline Chanul & Humiriastrun procerum & 3,0 & 3,45 & 2,56 \\
\hline Laurel & Cordia alliodora & 12,0 & 12,07 & 5,13 \\
\hline Chuncho & Cedrelinga cateneiformis & 6,0 & 5,17 & 2,56 \\
\hline Canelo & Ocotea sp. & 1,0 & 10,34 & \\
\hline Total & & 100,0 & 100,00 & 100,00 \\
\hline
\end{tabular}

Los resultados de las encuestas mostraron que los residuos de la especie eucalipto (Eucalyptus globulus) son los más utilizados en los tres cantones, valores que tienen relación con el primer lugar del volumen total de madera autorizada y aprovechada en el Ecuador que corresponde a las provincias de Cotopaxi y Chimborazo (MAE, 2010).

\section{Usos dados a los residuos}

El reporte de usos de los residuos indica que los clientes mayormente lo emplean para en producción avícola con el 48,39\% en el cantón Ambato y en menor cantidad para la producción de abono con el $2,86 \%$ en el cantón Riobamba (Tabla 11).

Tabla 11. Resumen porcentaje de usos que se dan a los residuos en cada cantón

\begin{tabular}{|c|c|c|c|c|c|c|c|}
\hline \multirow{2}{*}{ Cantón } & Caballerizas & Ladrilleras & Avícola & Abono & Energía & Tableros & Total \\
\cline { 2 - 7 } & $(\%)$ & $(\%)$ & $(\%)$ & $(\%)$ & $(\%)$ & $(\%)$ & $(\%)$ \\
\hline Riobamba & 11,43 & 21,43 & 28,57 & 2,86 & 7,14 & 28,57 & 100 \\
\hline Ambato & 0,00 & 19,35 & 48,39 & 12,90 & 0,00 & 19,35 & 100 \\
\hline Latacunga & 12,90 & 22,58 & 38,71 & 0,00 & 6,45 & 19,35 & 100 \\
\hline
\end{tabular}

El destino de los residuos que siguen luego de ser generados en el proceso de transformación de la madera identifica que están siendo comercializados de seis formas principales: la primera para la producción avícola que superan al $11 \%$ determinado en un estudio en Colombia donde el aserrín es utilizado en galpones; según Amaya (2015), la segunda es destinada 
a la producción de tableros, cuyas industrias pueden considerarse las consumidoras de residuos de madera por excelencia (Chacón, 2012).

En respuesta a la información analizada sobre los aserraderos, se tiene la ventaja de poder utilizar sus residuos para contribuir a cubrir una parte de las necesidades energéticas en aquellas industrias que posean hornos de secado de la madera (Saade et al., 2006).

\section{Conclusiones}

La principal especie utilizada en la producción de madera aserrada corresponde a Eucalyptus globulus o "eucalipto" con valores que fluctúan entre el $34-41.03 \%$, seguido por Pinus radiata o "pino" en los aserraderos de Riobamba y Latacunga, en el cantón Ambato presentó un comportamiento particular de uso, Hyeronima alchorneoides o "motilón", al resultar la segunda más utilizada con el $15.52 \%$, el resto se complementa con residuos de otras especies maderables.

El destino de los residuos generados demuestra que el nivel de gestión, se fundamenta en la comercialización para varios usos con un 80\%, seguido del autoconsumo con un $10 \%$, y en menor medida, se regala o acumula.

\section{Recomendaciones}

Evaluar de forma periódica el volumen y disponibilidad de residuos madereros tanto en las fases de transformación primaria y secundaria a fin de aprovechar las oportunidades para el desarrollo de nuevos emprendimientos.

Desarrollar estudios complementarios considerando el tipo de aserradero, tipos de residuos, volumen por especie y destino de los mismos para la utilización apropiada y reducción de riesgos ambientales.

\section{Agradecimiento}

A la empresa Novopan, S.A por la oportunidad que otorga a los profesionales y estudiantes de la Escuela de Ingeniería Forestal, Facultad de Recursos Naturales de la ESPOCH mediante convenios autorizados por el Máximo Organismo Institucional que permite llevar a cabo investigaciones, cuyos resultados contribuyen a la toma de decisiones para ejecutar acciones que mitiguen la contaminación ambiental.

\section{References:}

1. Amaya , C. (2015). Disposición de residuos industriales maderables: Una Estrategía Empresarial Ambiental para Convertir Costos en Inversiones. Tesis de pregrado, Universidad Militar de Nueva Granada, Colombia.

2. Balzarini, M. G.; Gonzalez, L.; Tablada, M., et. al. (2008). Infostat. Manual del Usuario. Córdova, Argentina: Brujas. 
3. Bequete, F. (1996). Un Mundo sin Derecho, una utopía? Revista Correos (XLIX): 43-45.

4. Castillo, R. (2007). Aprovechamiento de residuos de madera de Caoba y Manchiche para una concesión forestal del departamento del Petén. Tesis de pregrado, Facultad de Ingeniería, Universidad de San Carlos de Guatemala, Guatemala.

5. Chacón, L. (2012). Estudio: "Diagnóstico de las existencias de los residuos forestales en la región Huetar Norte de Costa Rica". San José: Ministerio de Ambiente y Energía. Fondo Nacional de Financiamiento Forestal.

6. FAO. (2014). El estado de los bosques en el mundo. Roma. Recuperado de: http://www.fao.org/3/a-i3710s.pdf

7. Kollmann, F. (2001). La promesa de tecnología. Trabajo presentado en VI Congreso Forestal Mundial por el Profesor Kollmann, de la Universidad de Munich, y presidente de la International Academy of wood Science.

8. MAE. Ministerio de Agricultura, Ganadería, Acuacultura y Pesca. (2010). Aprovechamiento de los Recursos Forestales 2007-2009. Quito, Ecuador.

9. MAE. (2011). Aprovechamiento de los Recursos Forestales 2010. Quito, Ecuador.

10. Manzanares, K., Velázques, D., y Guyat, M. A. (2007). Experiencias del aprovechamiento de los residuos en un aserrío. Ciencia e Investigación Forestal. 10. Instituto Forestal Chile e Instituto de Investigaciones Forestales/Cuba.

11. Pazmiño, I. (1997). Metodología de la Investigación Científica. Ecuador: Gráficas- Publicaciones

12. PRO ECUADOR. (2013). Muebles y productos de madera - Pro Ecuador. Recuperado de: http://www.proecuador.gob.ec/wpcontent/uploads/2015/06/Perfiles-de-Inversiones-Promocion-deInversiones/Perfiles-de-Inversion/Muebles-y-Productos-deMadera.pdf

13. Saade, L. C.; Venegas, J. M.; Bravo, K.; y Aguilera, A. (2006). Biomasa, un recurso energético para los aserraderos forestales. Michoacán: Coordinación de Ingeniería de Procesos Industriales y Ambientales del Instituto de Ingeniería de la UNAM.

14. Secretaría Nacional de Planificación y Desarrollo, S. (2013). Plan Nacional para el Buen Vivir 2013-2017, SENPLADES. Quito.

15. Uasuf, A., y Hilbert, J. (2012). El uso de la biomasa de Origen Forestal con destino a bioenergía en la Argentina. Instituto Nacional de Tecnología Agoprcuaria (INTA) en el marco del proyecto internacional BABETHANOL: 
16. Vignote, S., y Martínez, I. (2006). Tecnología de la madera ( $3^{\text {ra }}$ ed.). España: Mundi-Prensa.

Anexo1. Producción promedio de residuos forestales en los aserraderos cantón ${ }^{-1}$

\begin{tabular}{|c|c|c|c|c|c|}
\hline \multicolumn{2}{|c|}{ Riobamba } & \multicolumn{2}{|c|}{ Ambato } & \multicolumn{2}{|c|}{ Latacunga } \\
\hline $\begin{array}{c}\mathbf{N}^{\circ} \\
\text { aserradero }\end{array}$ & Producción (t) & $\begin{array}{c}\mathbf{N}^{\circ} \\
\text { aserradero }\end{array}$ & Producción $(t)$ & $\begin{array}{c}\mathbf{N}^{\circ} \\
\text { aserradero }\end{array}$ & Producción (t) \\
\hline 1 & 2 & 1 & 1 & 1 & 8 \\
\hline 2 & 1 & 2 & 1 & 2 & 2 \\
\hline 3 & 8 & 3 & 1 & 3 & 4 \\
\hline 4 & 4 & 4 & 1 & 4 & 4 \\
\hline 5 & 1 & 5 & 1 & 5 & 4 \\
\hline 6 & 12 & 6 & 2 & 6 & 1 \\
\hline 7 & 2 & 7 & 1 & 7 & 4 \\
\hline 8 & 1 & 8 & 1 & 8 & 4 \\
\hline 9 & 4 & 9 & 1 & 9 & 4 \\
\hline 10 & 2 & 10 & 1 & 10 & 1 \\
\hline 11 & 16 & 11 & 1 & 11 & 1 \\
\hline 12 & 1 & 12 & 1 & 12 & 1 \\
\hline 13 & 1 & 13 & 4 & 13 & 1 \\
\hline 14 & 1 & 14 & 4 & 14 & 1 \\
\hline 15 & 2 & 15 & 1 & 15 & 8 \\
\hline 16 & 4 & 16 & 4 & 16 & 1 \\
\hline 17 & 1 & 17 & 4 & 17 & 1 \\
\hline 18 & 2 & 18 & 1 & 18 & 1 \\
\hline 19 & 1 & 19 & 1 & 19 & 1 \\
\hline 20 & 1 & 20 & 1 & & \\
\hline 21 & 1 & 21 & 2 & & \\
\hline 22 & 1 & 22 & 1 & & \\
\hline 23 & 1 & 23 & 2 & & \\
\hline 24 & 1 & 24 & 2 & & \\
\hline 25 & 1 & 25 & 1 & & \\
\hline 26 & 2 & & & & \\
\hline 27 & 2 & & & & \\
\hline 28 & 3 & & & & \\
\hline 29 & 4 & & & & \\
\hline 30 & 2 & & & & \\
\hline 31 & 2 & & & & \\
\hline 32 & 1 & & & & \\
\hline 33 & 2 & & & & \\
\hline 34 & 4 & & & & \\
\hline 35 & 4 & & & & \\
\hline 36 & 1 & & & & \\
\hline 37 & 4 & & & & \\
\hline 38 & 8 & & & & \\
\hline Promedio & 2,92 & Promedio & 1,64 & Promedio & 2,74 \\
\hline
\end{tabular}

